\title{
Effect of HIV infection and antiretroviral therapy on immune cellular functions
}

\author{
Marek Korencak,, ${ }^{1}$ Morgan Byrne, ${ }^{2,3}$ Enrico Richter, ${ }^{1}$ Bruce T. Schultz,, Patrick Juszczak,, Julie A. Ake, \\ Anuradha Ganesan,, ${ }^{2,3}$ Jason F. Okulicz, ${ }^{2,5}$ Merlin L. Robb, ${ }^{3,4}$ Buena de los Reyes, ${ }^{6}$ Sandra Winning, ${ }^{6}$ \\ Joachim Fandrey, ${ }^{6}$ Timothy H. Burgess, ${ }^{2}$ Stefan Esser, ${ }^{7}$ Nelson L. Michael, ${ }^{4}$ Brian K. Agan,, ${ }^{2,3}$ \\ and Hendrik Streeck ${ }^{1}$ \\ IInstitute for HIV Research, University Hospital, University Duisburg-Essen, Essen, Germany. ${ }^{2}$ Infectious Disease Clinical \\ Research Program, Department of Preventive Medicine and Biostatistics, Uniformed Services University of the Health \\ Sciences, Bethesda, Maryland, USA. ${ }^{3}$ Henry M. Jackson Foundation for the Advancement of Military Medicine, Bethesda, \\ Maryland, USA. ${ }^{4}$ United States Military HIV Research Program, Walter Reed Army Institute of Research, Silver Spring, \\ Maryland, USA. ${ }^{5}$ Infectious Disease Service, San Antonio Military Medical Center, Fort Sam Houston, Texas, USA. \\ ${ }^{6}$ Institute for Physiology, University Duisburg-Essen, Essen, Germany. ${ }^{7}$ HPSTD HIV Clinic, University Hospital, University \\ Duisburg-Essen, Essen, Germany.
}

During chronic HIV infection, immune cells become increasingly dysfunctional and exhausted. Little is known about how immune functions are restored after initiation of antiretroviral therapy (ART). In this study, we assessed cellular and metabolic activity and evaluated the effect of individual antiretrovirals on cellular subsets ex vivo in ART-treated and treatment-naive chronically HIV-infected individuals. We observed that cellular respiration was significantly decreased in most immune cells in chronic HIV infection. The respiration was correlated to immune activation and the inhibitory receptor programmed cell death 1 on CD8 ${ }^{+}$T cells. ART restored the metabolic phenotype, but the respiratory impairment persisted in $\mathrm{CD}^{+} \mathrm{T}$ cells. This was particularly the case for individuals receiving integrase strand transfer inhibitors. CD4 ${ }^{+} \mathrm{T}$ cells from these individuals showed a significant reduction in ex vivo proliferative capacity compared with individuals treated with protease inhibitors or nonnucleoside reverse transcriptase inhibitors. We noticed a significant decrease in respiration of cells treated with dolutegravir (DLG) or elvitegravir (EVG) and a switch from polyfunctional to TNF- $\alpha$-dominated "stress" immune response. There was no effect on glycolysis, consistent with impaired mitochondrial function. We detected increased levels of mitochondrial ROS and mitochondrial mass. These findings indicate that EVC and DLC use is associated with slow proliferation and impaired respiration with underlying mitochondrial dysfunction, resulting in overall decreased cellular function in $\mathrm{CD}^{+} \mathrm{T}$ cells.

Conflict of interest: The authors have declared that no conflict of interest exists.

Copyright: (c) 2019 American Society for Clinical Investigation

Submitted: December 13, 2018

Accepted: May 10, 2019

Published: June 20, 2019

Reference information: /Cl Insight. 2019;4(12):e126675. https://doi. org/10.1172/jici.nsight.126675

\section{Introduction}

Chronic HIV infection results in progressive exhaustion of the immune system (1), which is characterized by a reduction in antiviral effector activities, such as cytokine secretion, proliferation, and cytotoxicity, necessary to control viral replication. Immune system exhaustion is further characterized by an increase of inhibitory receptor expression on the cell surface, in particular on T cells, which attenuate cellular activities (2). The reduction of immune cellular function has been demonstrated to be linked to a reduction in cellular metabolism. For example, the ability of T cells to produce IFN- $\gamma$ is significantly decreased when blocking aerobic glycolysis (3). Furthermore, immune cells exposed to glycolytic or fatty acid synthase inhibitors have perturbed metabolic alterations and display massive reduction in cytokine production (4).

Although this is not surprising, because cellular metabolism is the underlying mechanism for a cell to gain energy and more active cells have higher metabolism, it appears also to be a system to control immunological pathways. Metabolism controls action, survival, and proliferation of cells (5). Upon activation, immune cells undergo several changes, including metabolic reprograming to provide adenosine triphosphate (ATP) and intermediates for cell growth (6). Activated T cells ferment glucose into lactate in the presence of oxygen to support mitochondrial oxidative phosphorylation (OXPHOS), also known as aerobic glycolysis or Warburg effect. OXPHOS is a metabolic pathway that is used to oxidize different nutri- 
ents to produce energy in the form of ATP and compared with glycolysis produces higher yield of ATP. Although OXPHOS occurs mostly in resting or memory lymphocytes, glycolysis provides intermediates for cell growth and proliferation and occurs in activated or effector cells (7). During activation, T cells express inhibitory receptors that control autoreactivity and immunopathology (8); however, higher and sustained expression of these molecules is a sign of $\mathrm{T}$ cell exhaustion. Inhibitory receptors themselves seem to actively interfere with metabolism as has been demonstrated. Ligation of the inhibitory receptor programmed cell death 1 (PD-1) can reduce T cell effector function (9). Moreover, expression of PD-1 inhibits uptake of glucose and glutamine and shifts the metabolism toward fatty acid $\beta$-oxidation (10).

The progression of exhaustion and immune dysfunction in chronic HIV infection can be halted, and even reverted, with the initiation of antiretroviral therapy (ART; ref. 11). To date, several classes of antiretroviral medication with distinct mechanisms have been discovered. Common ART regimens use a combination of 3 antiretroviral agents with 2 modes of actions (12). Nucleoside reverse transcriptase inhibitors (NRTIs) and nonnucleoside reverse transcriptase inhibitors (NNRTIs) block viral RNA transcription by inhibiting reverse transcriptase, protease inhibitors (PIs) block viral enzyme protease and prevent the maturation of the virus, and integrase inhibitors act against the viral DNA integration into the host genome (13). The newest class represents the integrase strand transfer inhibitors (INSTIs) - raltegravir (RAL), elvitegravir (EVG), and dolutegravir (DLG). Because INSTIs specifically block the process of viral DNA integration into the host genome by occupying the active site of integrase, their effects are thought to be highly specific (14). Given the superior profile of INSTIs, including reduced potential for drug or food interactions, similar or better tolerability and safety, while showing noninferiority in viral suppression (15-18), INSTI-based regimens have been recommended as initial therapy for many people diagnosed with HIV (19).

Although ART can successfully suppress viral replication, long-term toxicity of some antiretroviral medications has been a serious issue that may compromise treatment effectiveness (20). Although the newer generation of antiretroviral medications and in particular INSTIs are believed to commonly manifest only minor side effects, older ARTs have demonstrated severe long-term toxicity, such as NRTI-related mitochondrial toxicity (21). It has been suggested that some NRTIs cause oxidative stress on cells, defined as an imbalance between the production of ROS and the cellular antioxidant defenses that prevent damage from those species. Oxidative stress might be a principal event in the toxicity of some NRTIs and could cause defects in energetics and mitochondrial DNA (mtDNA) replication. Indeed, it has been shown that there are major differences in mitochondrial mass between HIV-negative and HIV-positive ART-treated individuals (22). In some cases, this manifests as serious side effects, such as hepatic failure, lipodystrophy, and lactic acidosis. ART has been also associated to changes in metabolism, affecting metabolism of lipids and glucose homeostasis $(23,24)$. The effect of INSTIs on mitochondrial function has not been systematically assessed, but there are limited data suggesting that this may be a concern (25).

In this study, we assessed cellular and metabolic activity and effect of individual ART regimens on immune cellular subsets ex vivo in ART-treated and chronically HIV-infected, treatment-naive individuals. We show that the respiration of immune cells in chronic HIV infection is significantly impaired and this is linked to overall chronic immune activation and exhaustion. We demonstrate that ART is able to restore original metabolic profiles but not in case of $\mathrm{CD}^{+} \mathrm{T}$ cells, in particular INSTI-containing regimens. We found a significant interference of INSTIs with $\mathrm{CD}^{+} \mathrm{T}$ cell respiration, proliferation, and immune responses, resulting in decreased immune cellular function.

\section{Results}

To understand changes in immune metabolism in subjects with chronic HIV following immunological restoration by ART, we cross-sectionally assessed differences in the immune metabolism of different cell subsets. We first determined differences in 2 major metabolic pathways - OXPHOS and glycolysis. Mitochondrial respiration was assessed by measuring oxygen consumption rate (OCR) and aerobic glycolysis by measuring the acidification of the medium (extracellular acidification rate [ECAR]) using an extracellular flux analyzer (Seahorse $\mathrm{XFe}$ ) in $\mathrm{CD}^{+}$and $\mathrm{CD}^{+} \mathrm{T}$ cells, B cells, and NK cells of HIV-negative individuals. We observed that all cellular subsets have significantly different respiratory and glycolytic activity, suggesting differential demand for oxygen and glucose to maintain their vitality (Supplemental Figure 1; supplemental material available online with this article; https://doi.org/10.1172/jci.insight.126675DS1). Interestingly, CD4 ${ }^{+} \mathrm{T}$ cells 
showed the highest metabolic activity and were even significantly higher compared with their $\mathrm{CD}^{+} \mathrm{T}$ cell counterpart. Furthermore, the bioenergetic profile of $\mathrm{CD}^{+}$and $\mathrm{CD} 8^{+} \mathrm{T}$ cells was significantly higher compared with B cells. NK cells showed, overall, the lowest activity in HIV-negative individuals.

We next compared respiration and glycolysis of HIV-infected, treatment-naive individuals $(n=11)$ and healthy controls $(n=12)$. Both basal and maximal respiration of T cells and NK cells appeared to be significantly impaired in HIV-infected, treatment-naive individuals (Figure 1A). In addition to decreased respiration, NK cells, but not T cells and B cells, showed reduced glycolysis and glycolytic capacity (Figure 1B). The difference in glycolysis was less significant compared with changes observed in respiration, indicating a shift in the bioenergetic profile of lymphocytes toward glycolysis during HIV infection. The OCR/ECAR ratio shows the relative contribution of OXPHOS and glycolysis to energy production. Indeed, we found a significant overall difference in T cell profiles in HIV-infected, treatment-naive individuals by OCR/ECAR ratio (Figure 1C). A higher OCR/ ECAR ratio in HIV-negative T cells indicates that the energy is mainly produced by OXPHOS, whereas the lower OCR/ECAR ratio in HIV-positive, treatment-naive T cells indicates a shift to glycolysis and chronic immune activation of these cells caused by the presence of HIV. Thus, our data demonstrate noticeable differences in the metabolic profile of HIV-infected individuals that chronic activation might cause.

We therefore investigated whether the bioenergetic profile differences are associated with chronic immune activation, measured by CD38 and HLA-DR expression on CD8 ${ }^{+} \mathrm{T}$ cells (26). As expected, we found a strong correlation between chronic immune activation and basal respiration, maximal respiration, glycolysis, and maximal glycolysis, linking the $\mathrm{CD} 8^{+} \mathrm{T}$ cell activation phenotype to the bioenergetic profile (Figure 1D). Increased percentage of chronically activated cells decreased the respiratory activity or glycolysis. Interestingly, we did not observe the same association of $\mathrm{CD}^{+} \mathrm{T}$ cell activation and metabolic profile (Supplemental Figure 2), consistent with chronic immune activation but not the activation of $\mathrm{T}$ cells. We next hypothesized that increased expression of PD-1, a prominent marker for $\mathrm{T}$ cell exhaustion (27), is associated with metabolic alteration and overall decreased metabolic activity (Figure 1E). Similar to observations in the lymphochoriomeningitis virus infection mouse model (28), we found a strong association between $\mathrm{T}$ cell phenotypic exhaustion of $\mathrm{CD} 8^{+} \mathrm{T}$ cells with all metabolic parameters, confirming that the PD-1 signaling not only inhibits effector functions, as previously demonstrated, but also affects cellular metabolism. Taken together, the data suggest that chronic immune activation and $\mathrm{T}$ cell dysfunction is directly linked to the impaired metabolic profiles observed in chronic HIV infection.

We next sought to determine whether ART and full suppression of viremia can restore metabolic profiles. We expected an improvement in all metabolic parameters of all cell types because previous work demonstrated that ART reduces overall immune activation and reverts exhaustion phenotypes $(1,2)$. Indeed, NK cells, B cells, and $C D 8^{+} \mathrm{T}$ cells were able to restore their metabolic profiles after an average of 6.4 years on treatment (Supplemental Figure 3). However, we observed that the basal respiration and maximal respiratory capacity of $\mathrm{CD}^{+} \mathrm{T}$ cells in $\mathrm{HIV}$-treated individuals remained significantly decreased compared with healthy controls $(P>0.001$ for basal respiration, and $P<0.05$ for maximal respiratory capacity) (Figure 2A), without an effect on glycolysis (Figure 2B). Moreover, we not only found a lack of restoration in the respiratory profile but also found a partial shutdown of $\mathrm{CD}^{+} \mathrm{T}$ cell respiratory activity and shift toward glycolysis, as indicated by the OCR/ECAR ratio of $\mathrm{CD}^{+} \mathrm{T}$ cells (Figure 2, C and D). To understand the cause for a decrease in respiratory activity of $\mathrm{CD}^{+} \mathrm{T}$ cells, we first stratified all individuals by treatment regimens (Supplemental Table 1). Interestingly, DLG as part of a triple-therapy regimen showed consistently lower basal and maximal respiration compared with the other non-INSTI ART regimens (Figure 2E). We next assessed whether the reduction in respiratory capacity of $\mathrm{CD}^{+} \mathrm{T}$ cells in the INSTI-containing regimens was resulting in reduced $\mathrm{CD}^{+} \mathrm{T}$ cell proliferation. We therefore performed ex vivo proliferation studies with 54 participants (Supplemental Table 2, A and B) who received an INSTI-, NNRTI-, or PI-containing ART regimen and had fully suppressed viremia for a period longer than 6 months. We observed that cells from individuals receiving INSTI showed significantly lower ex vivo proliferation compared with $\mathrm{CD} 4^{+} \mathrm{T}$ cells from individuals receiving either PI- or NNRTI-containing regimens (Figure 2F). Thus, these data suggest reduced $\mathrm{CD}^{+} \mathrm{T}$ cell proliferation in individuals receiving INSTI-containing regimens.

To dissect the effect of individual antiretroviral medications, $\mathrm{CD}^{+} \mathrm{T}$ cells from HIV-negative, healthy individuals were exposed to antiretroviral medication at previously described bioactive plasma concentrations (as described in Supplemental Table 3). In addition, the biological antiviral activity of DLG, EVG, RAL, and tenofovir disoproxil fumarate (TDF) was confirmed in vitro (Supplemental Figure 4). 
A
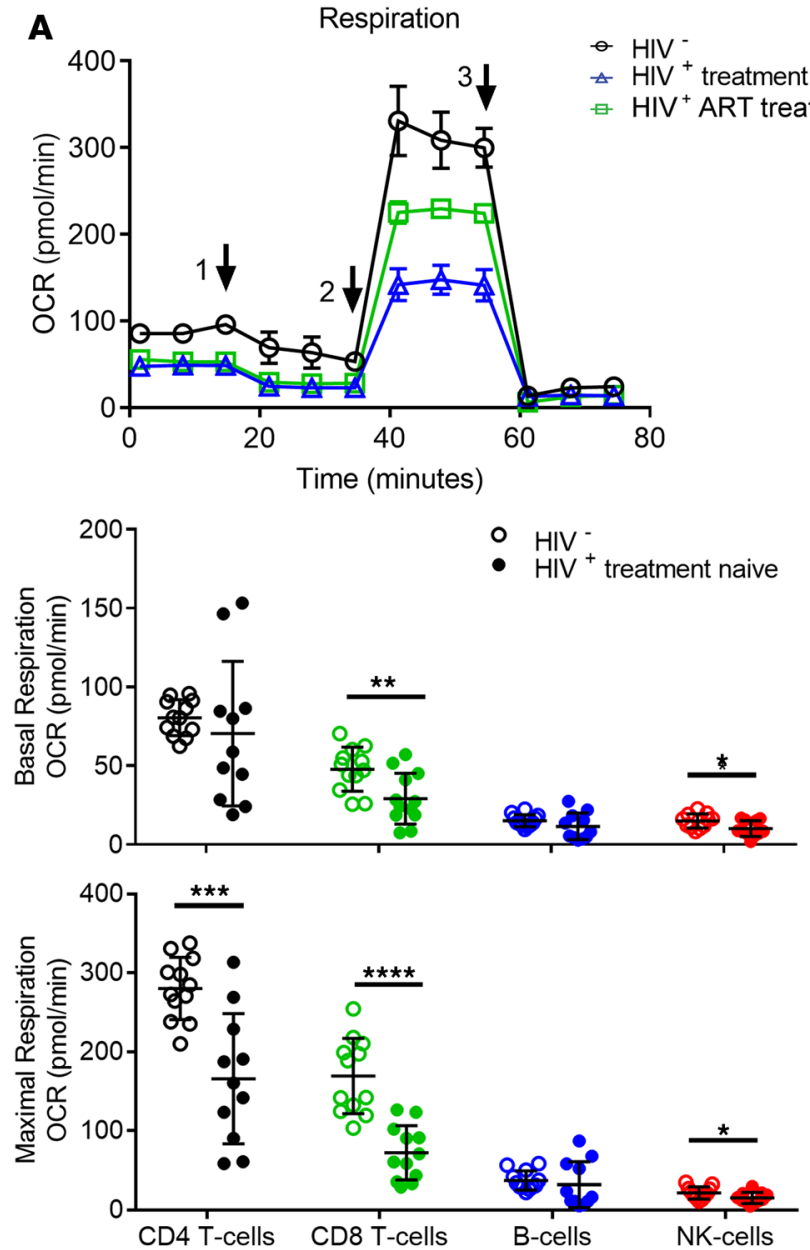

C

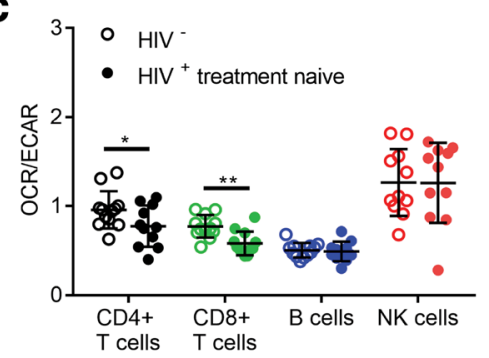

D
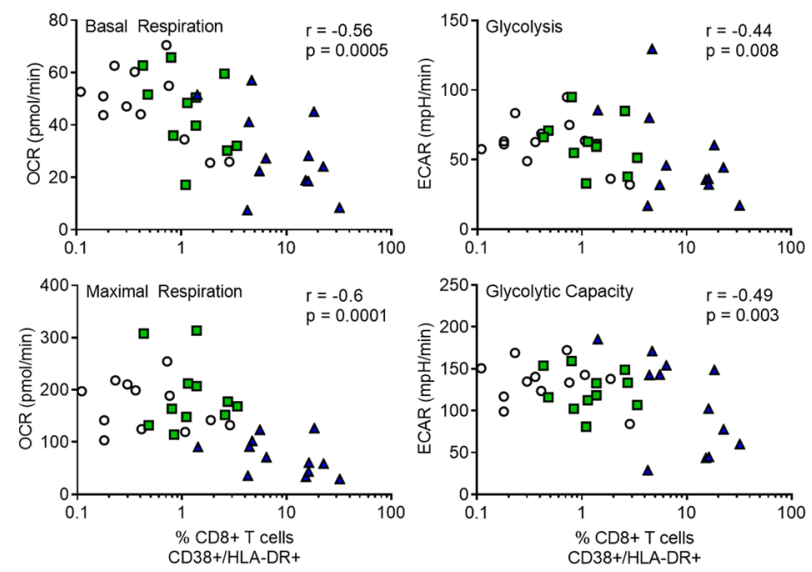
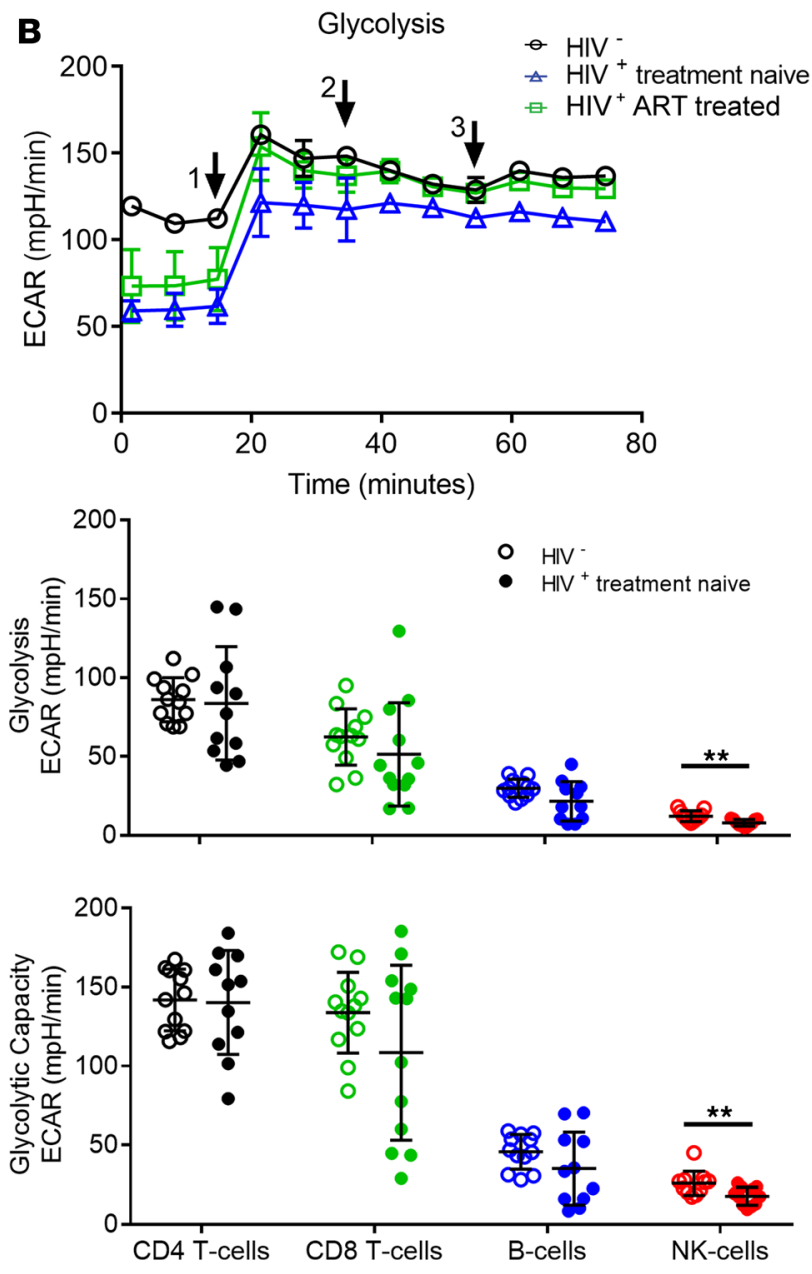

${ }^{\circ} \mathrm{HIV}^{-} \triangle \mathrm{HIV}^{+}$treatment naive
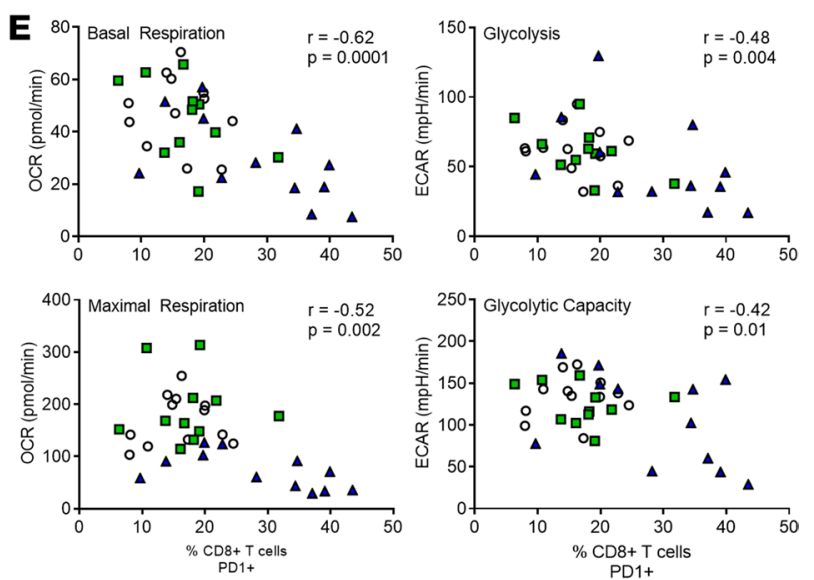
Figure 1. CD4 ${ }^{+}$and CD8 ${ }^{+}$T cells and NK cells from HIV-positive, treatment-naive patients $(n=11)$ show reduced basal and maximal respiration compared with HIV-negative patients $(\boldsymbol{n}=\mathbf{1 2})$. (A) Representative plot of oxidative consumption rate (OCR) showing subsequent injections of test components (marked with arrowheads) (1) oligomycin, (2) FCCP, and (3) rotenone with antimycin A. Comparison of basal and maximal mitochondrial respiration between HIV-positive, treatment-naive individuals and healthy controls demonstrating reduced basal and maximal respiration of CD4 ${ }^{+}$and $\mathrm{CD} 8^{+} \mathrm{T}$ cells and NK cells in HIV-positive, treatment-naive individuals. (B) Representative plot of extracellular acidification rate (ECAR) with the same injection strategy as the previous test. No significant differences in glycolysis and glycolytic capacity were observed among CD4+ and CD8+ T cells and NK cells. (C) OCR/ECAR ratio showing differences in the bioenergetic profiles. (D) Negative correlation between activation (characterized as CD38+ $\mathrm{HLA}^{-\mathrm{DR}} \mathrm{R}^{+}$) and metabolism in CD8 ${ }^{+} T$ cells. (E) Negative correlation between exhaustion (characterized as PD-1+) and metabolic parameters in CD8 ${ }^{+} \mathrm{T}$ cells. Plots show individual values with the mean \pm SD. Bar chart shows the mean value \pm SD. Statistical significance was assessed by Kruskal-Wallis test with Dunn's multiple-comparisons test and repeated-measures (RM) 1-way ANOVA test with Holm-Šídák's multiple-comparisons test $\left({ }^{*} P<0.05 ;^{* *} P<0.01 ;{ }^{* *} P<0.001\right.$; $\left.{ }^{* * * *} P<0.0001\right)$.

Most of the antiretroviral compounds had no effect on metabolic functions of CD4+ $\mathrm{T}$ cells (Figure 3, A and B). However, both INSTIs DLG and EVG, but not RAL, significantly reduced both basal and maximal respiration, with no effect on cellular glycolysis measured by Seahorse (Supplemental Figure 5) and confirmed by Western blot analysis of HIF-1 $\alpha$ and expression of HIF-1 target genes Glut1 and PKG1, involved in glycolysis (Figure 3C), therefore suggesting that both INSTIs specifically interfere with the mitochondrial respiration pathway. However, no changes in mitochondrial membrane potential in cells treated with INSTIs were observed (Figure 3D). A dose dependency of INSTI-mediated respiratory reduction was observed along with almost complete shutdown of cellular respiration at 5-fold plasma concentration, while other compounds displayed no effect on cellular respiration at this concentration (Supplemental Figure 6). The observed impact was not due to potential cellular cytotoxicity demonstrating stable viability over the same period (Supplemental Figure 7). Additionally, we performed titration of DLG, and we identified that the first lowest concentration, which had no effect on respiration, was 16-fold lower than plasma concentration (Supplemental Figure 8).

Previous studies suggested that multifunctional $\mathrm{CD}^{+} \mathrm{T}$ cell immune responses have intrinsic superiority in their anti-HIV immune response and provide better help for $\mathrm{CD}^{+} \mathrm{T}$ cells to clear viral infection (29-31). We analyzed the multifunctional profile of $\mathrm{CD}^{+} \mathrm{T}$ cells pretreated with antiretroviral compounds using a 5-marker functional panel after superantigen staphylococcal enterotoxin B (SEB) stimulation (gating strategy shown in Supplemental Figure 9). Cells stimulated in the presence of DLG or EVG but not any other antiretroviral medication demonstrated a significant decrease in the overall immune response (Figure 3, E and F). More strikingly, both drugs changed the former multifunctional response to a monofunctional response. One of the most notable differences was the decrease of immunomodulatory cytokines in the absence of significant changes to the TNF- $\alpha$ stress immune response, indicating a change in favor of the immune response toward a functional stress phenotype in the presence of EVG or DLG.

EVG is structurally similar to quinolone antibiotics (32), of which some have known mitochondrial toxicity (33). Given the stress signature of the immune responses of $\mathrm{CD}^{+} \mathrm{T}$ cells and changes in the basal and maximal respiration of $\mathrm{CD}^{+} \mathrm{T}$ cells in the presence of DLG and EVG, we hypothesized that both drugs interfere with mitochondrial metabolism. It has been previously demonstrated that NRTIs can block polymerase- $\gamma$ in mitochondria, thus leading to a decrease in mtDNA copy numbers (34). The mtDNA levels in cells were therefore measured, and mtDNA levels after treatment with both EVG and DLG INSTIs and also with the PI RTV were increased (Figure 4A). Interestingly, it has been previously shown that increased mtDNA can be a result of transmembrane potential $(\Delta \psi \mathrm{m})$ loss through interference in the electron transportation chain (ETC) during OXPHOS. Because this interference also would increase measurable ROS, any antiretroviral medication-associated increases in intracellular ROS levels were assessed. EVG, DLG, and RTV significantly increased mitochondrial ROS content in CD4 ${ }^{+} \mathrm{T}$ cells (Figure 4B), demonstrating the interference of INSTIs in the ETC during OXPHOS.

\section{Discussion}

Although HIV infection is evolving into a chronic condition with comparable life expectancy to the uninfected population in patients who are adherent to ART, there is evidence for increased risk of cardiovascular diseases as well as cancer even in well-ART-suppressed individuals. However, the underlying mechanism for this phenomenon is unknown. Research into the mechanisms behind cellular functions and chronic immune activation or metabolism may provide targets for future interventions. In this study we assessed the effect of chronic HIV viremia and ART on immune metabolism. Among the peripheral immune cells that we studied, we observed that $\mathrm{CD} 4^{+} \mathrm{T}$ cells were metabolically most active. We further discovered that some 


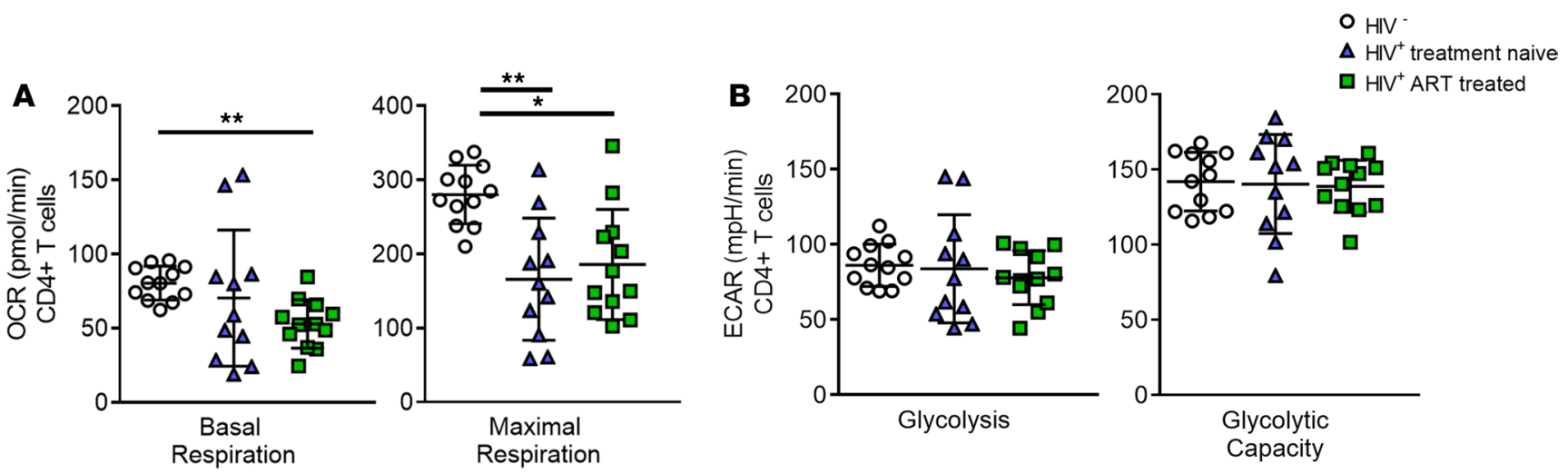

${ }^{\circ} \mathrm{HIV}^{-} \quad \Delta \mathrm{HIV}^{+}$treatment naive $\quad \mathrm{HIV}^{+} \mathrm{ART}$ treated

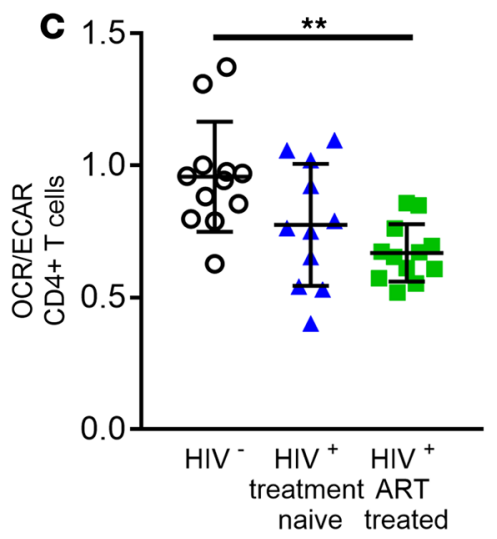

D

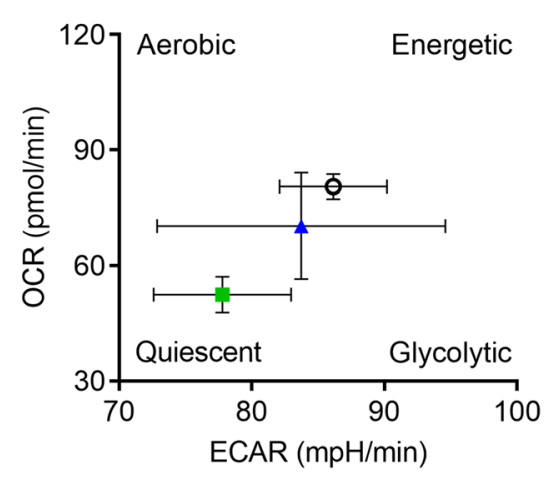

$\mathbf{E}$

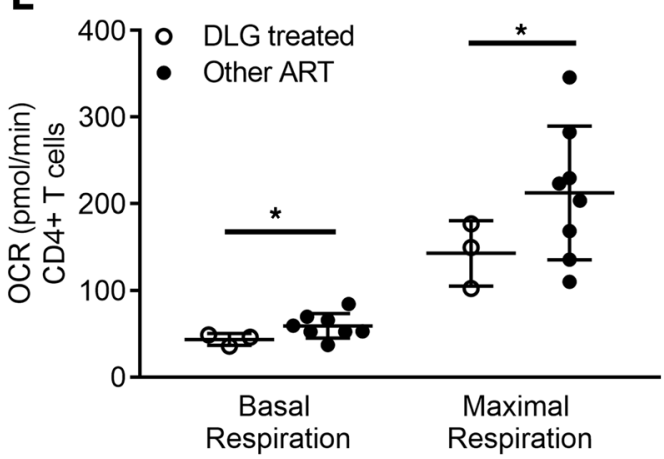

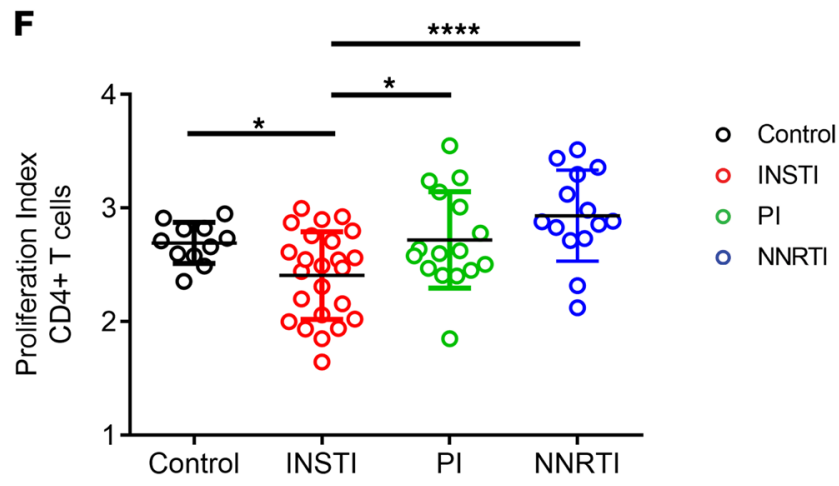

Figure 2. CD4 ${ }^{+}$T cells from HIV-positive, treatment-naive $(n=11)$ and ART-treated individuals $(n=12)$ display reduced basal and maximal respiration compared with HIV-negative individuals $(\boldsymbol{n}=\mathbf{1 2})$. (A) Comparison of basal and maximal mitochondrial respiration between HIV-positive treatment-naive and treated individuals and healthy controls demonstrating reduced basal and maximal respiration of CD4 ${ }^{+} T$ cells in ART-treated individuals. (B) No significant differences in glycolysis and glycolytic capacity of CD4+ $T$ cells in the 3 groups. (C and D) Schematic graphs of bioenergetic profiles of ART-treated, chronically untreated, and healthy individuals, as shown in differences in OCR/ECAR ratio highlighting differences in the bioenergetics profiles between the 3 groups. (E) Decreased OXPHOS of ART-treated, HIV-positive individuals receiving a DLG-containing regimen (open circles) compared with PI-containing, NNRTI-containing (black circles). (F) Significantly decreased proliferation of CD4 ${ }^{+}$T cells of ART-treated, HIV-positive individuals receiving an INSTI-containing regimen compared with PI-containing and NNRTI-containing regimens. Bar chart shows the mean value \pm SD. Statistical significance was assessed by Kruskal-Wallis test with Dunn's multiple-comparisons test and RM 1-way ANOVA test with Holm-Šídák's multiple-comparisons test $\left({ }^{*} P<0.05 ;{ }^{* *} P<0.01 ;{ }^{* * *} P<0.0001\right)$.

ARVs, in particular the newer INSTIs (DLG and EVG), had a significant effect on CD4 $4^{+} \mathrm{T}$ cell respiratory activity. We demonstrate that these INSTIs interfere in the ETC of mitochondria, severely impairing the respiratory capacity of $\mathrm{CD} 4^{+} \mathrm{T}$ cells. Further, changes in $\mathrm{CD} 4^{+} \mathrm{T}$ cell respiration lead to a skewing of $\mathrm{CD} 4^{+}$ $\mathrm{T}$ cell immune responses toward a reduced and monofunctional $\mathrm{CD} 4$ response, increased ROS production, and increased cytoplasmatic mtDNA indicative of a leaky mitochondrial membrane. 

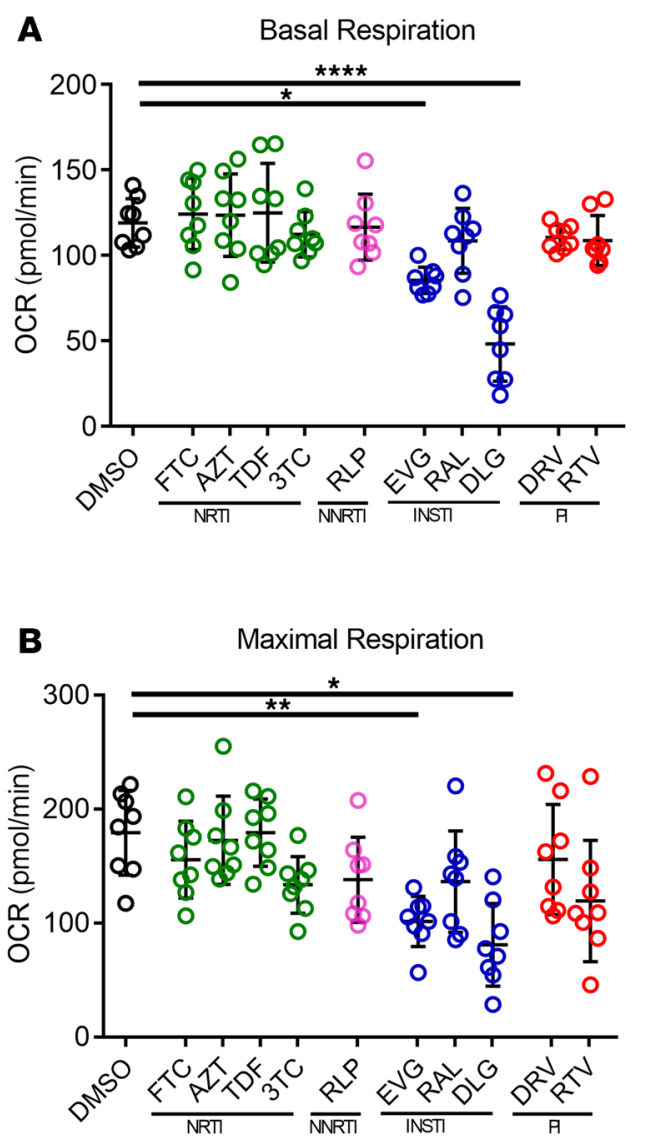

E

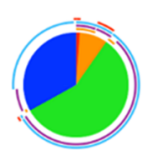

DMSO
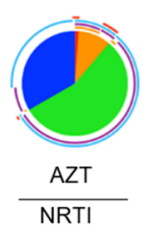
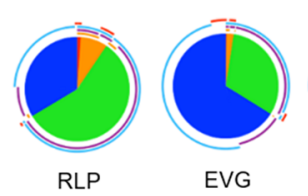
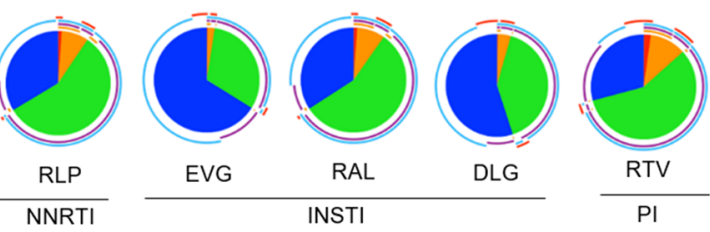

$$
\begin{gathered}
\text { Number of } \nabla 2 \\
\text { cytokines } \nabla 3
\end{gathered}
$$

$$
\checkmark 4
$$

RAL

DLG

$\mathbf{F}$
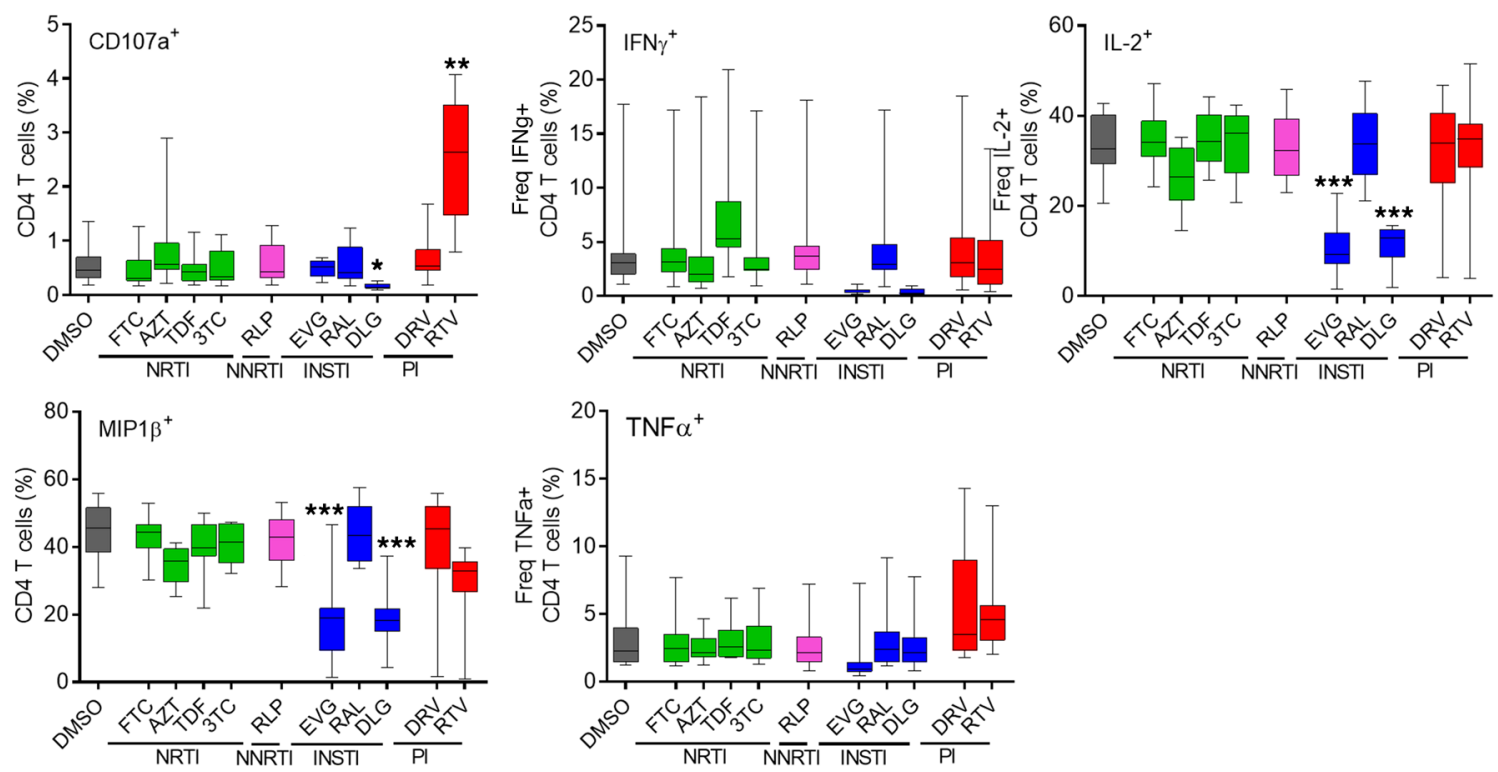
Figure 3. Assessment of effect of ARTs on $\mathrm{CD4}^{+} \mathbf{T}$ cell metabolism and functionality $(\boldsymbol{n}=\mathbf{8})$. Basal $(\mathbf{A})$ and maximal respiration $(\mathbf{B})$ of CD4 ${ }^{+} \mathrm{T}$ cells exposed to different ARTs for 3 days. Significant reduction in OCR was observed for CD4+ T cells treated with DLG and EVG. FTC, emtricitabine; AZT, zidovudine; 3TC, lamivudine; RLP, rilpivirine; DRV, darunavir ethanolate; RTV, ritonavir; R, Ruskinn Invivo Hypoxia Workstation; HOX, hypoxic condition. (C) HIF-1 $\alpha$ protein expression and Glut1 and PGK1 mRNA levels of cells treated with DMSO, NRTIs (represented by FTC), NNRTIs (represented by RLP), ISNTIs (represented by DLC), and PIs (represented by DRV) showing no significant differences. (D) Membrane potential without and with mitochondrial uncoupler FCCP. (E) Assessment of $C D 4^{+} T$ cell polyfunctionality after stimulation with SEB in the presence of different ARTs. Overall reduced functionality was observed in the presence of DLG and EVG for CD107a, IFN- $\gamma$, IL-2, macrophage inflammatory protein-1 $\beta$ (MIP-1 $\beta$ ), and TNF- $\alpha$. (F) SPICE analysis showing a shift in the polyfunctionality profile of $C D 4^{+} T$ cells stimulated in the presence of DLG and EVG toward a TNF- $\alpha$-dominated, monofunctional response. Plots show individual values with the mean $\pm \mathrm{SD}$. Box plots show the median value within fifth to 95th percentile. Statistical significance was assessed by RM 1-way ANOVA test with Holm-Šídák's multiple-comparisons test $\left({ }^{*} P<0.05 ;{ }^{* *} P<0.01 ;{ }^{* * *} P<0.001 ;{ }^{* * *} P<0.0001\right)$.

Changes in metabolism of $\mathrm{CD}^{+}$and $\mathrm{CD}^{+} \mathrm{T}$ cells have been observed in previous studies (35); however, overall comparison of metabolic profiles within lymphocytes has not been studied. Given the different roles in the immune system, we observed that $\mathrm{CD} 4^{+} \mathrm{T}$ cells, $\mathrm{CD} 8^{+} \mathrm{T}$ cells, $\mathrm{B}$ cells, and NK cells have significantly different demands on oxygen, resulting in different levels of OXPHOS. Some studies have already demonstrated that HIV can cause changes in cellular metabolism in different parts of the immune system. For example, both $\mathrm{CD} 4^{+} \mathrm{T}$ and $\mathrm{CD} 8^{+} \mathrm{T}$ cells increase expression of Glut1 during chronic HIV infection as well as their mitochondrial mass $(36,37)$. When comparing metabolic profiles of chronically HIV-infected, treatment-naive individuals and matched healthy controls, we observed massive reduction in respiration in HIV-positive individuals. Changes in glycolysis were also previously described (38); however, the driving force of these changes is still not clear. $\mathrm{CD} 4^{+}$and $\mathrm{CD} 8^{+} \mathrm{T}$ cells, B cells, and NK cells isolated from HIV-infected, treatment-naive individuals showed significantly lower basal and maximal respiration. Additionally, we found a link between the chronic immune activation of $\mathrm{CD}^{+} \mathrm{T}$ cells and their metabolism.

Although previous studies have demonstrated a restoration of $\mathrm{CD} 4^{+}$and $\mathrm{CD} 8^{+} \mathrm{T}$ cell function with the initiation of ART (39-42), we observed, contrary to this, a reduction in basal and maximal respiration after initiation of ART in $\mathrm{CD}^{+} \mathrm{T}$ cells. Combination ART regimens have evolved to the use of several classes of drugs with greater potency, safety, and convenience (43). The ultimate goal of ART is the preservation and recovery of immune health. INSTIs have become part of first-line ART regimens, with faster CD4 ${ }^{+} \mathrm{T}$ cell recovery than with other ART regimens (44). The data presented here identify a potentially previously unknown effect of INSTIs on $\mathrm{CD} 4^{+} \mathrm{T}$ cell metabolism and function. Pathological changes in $\mathrm{CD} 4^{+} \mathrm{T}$ cell metabolism and dysregulated functional responses were seen with INSTI exposure in both ex vivo and in vitro experiments. Although $\mathrm{CD}^{+} \mathrm{T}$ cells, NK cells, and $\mathrm{B}$ cells were able to restore their metabolic capacity after ART treatment, the respiration of $\mathrm{CD}^{+} \mathrm{T}$ cells remained significantly decreased, in particular in INSTI-containing regimens. We demonstrated significantly impaired CD4 ${ }^{+} \mathrm{T}$ cell mitochondrial respiration in 2 INSTIs - DLG and EVG. Mitochondrial toxicity and changes in mitochondrial turnover have been already described for NRTIs but not INSTIs (45). NRTIs, particularly thymidine analogs, appear to inhibit DNA polymerase- $\gamma$, a mitochondrial enzyme responsible for mtDNA replication (46), leading to mitochondrial toxicity. Other suggested mechanisms of NRTI-induced mitochondrial toxicity include the inhibition of endogenous nucleotide kinase, the generation of ROS, mutations in nuclear and mitochondrial DNA, and changes in expression of uncoupling proteins (47). Our data show changes in both mtDNA and generation of ROS, suggesting that the mechanism of mitochondrial toxicity observed in INSTIs might share the same or similar pathways observed in NRTI-related mitochondrial toxicity.

The data presented here indicate that DLG and EVG had the greatest negative effect on $\mathrm{CD}^{+} \mathrm{T}$ cell respiration. Biologically, the effect of INSTIs on mitochondria is not necessarily surprising. EVG shares structural similarity to quinolone antibiotics (32). It has been demonstrated that quinolone antibiotics can interfere with the ETC during OXPHOS, thereby reducing basal and maximal respiration (48). The data here show EVG and DLG have substantial mitochondrial toxicity ex vivo with significantly increased ROS production and an increase in mtDNA. Both are signs of mitochondrial damage and interference in the ETC during OXPHOS. These findings are also compatible with the observation of reduced basal and maximal respiration. It is likely, however, that the observed effect is systemic and not only restricted to CD4 ${ }^{+} \mathrm{T}$ cells. Indeed, in this study, we found that $\mathrm{CD} 4^{+} \mathrm{T}$ cells were metabolically most active compared with the all other immune cells, and therefore subtle differences became more visible in this subset compared with others.

Besides causing changes in $\mathrm{CD}^{+} \mathrm{T}$ cell metabolism, DLG and EVG degrade the functional response of $\mathrm{CD}^{+} \mathrm{T}$ cells. When we used SEB stimulation as a surrogate for optimal $\mathrm{T}$ cell receptor-triggered (TCR-triggered) stimulation, $\mathrm{CD}^{+} \mathrm{T}$ cell responses were not only significantly reduced but also shift- 
A

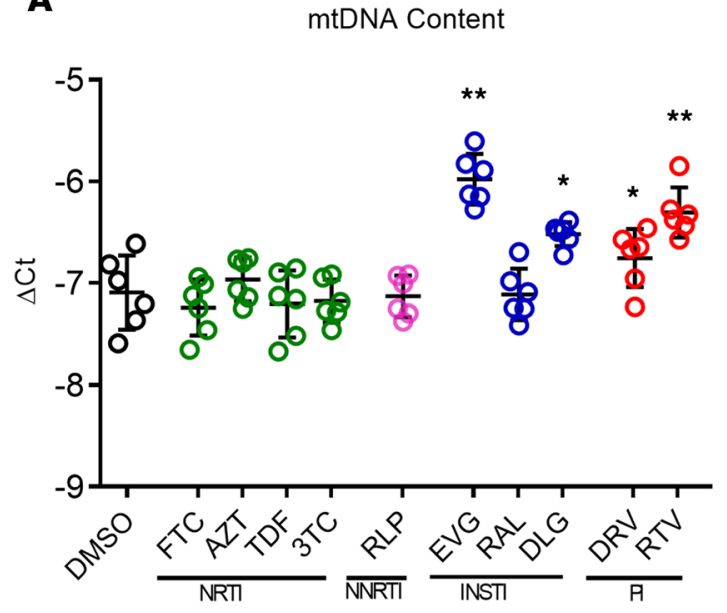

B

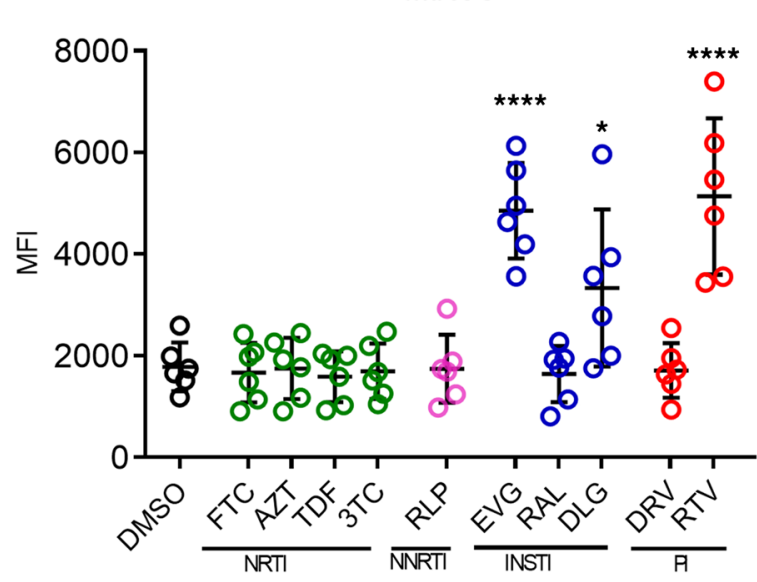

Figure 4. Shift in mitochondrial ROS production and mitochondrial content (measured as amount of mtDNA) after ART exposure for 3 days $(n=6)$. (A) $\mathrm{CD4}^{+}$T cells exposed to DLG, EVG, and RTV showed increased production of mitochondrial ROS (mtROS). (B) Increased mtDNA content of CD4 ${ }^{+}$cells upon incubation with DLG, EVG, and RTV for 3 days. Plots show individual values with the mean \pm SD. Statistical significance was assessed by RM 1-way ANOVA test with Holm-Šídák's multiple-comparisons test $\left({ }^{*} P<0.05 ;{ }^{* *} P<0.01 ;{ }^{* * *} P<0.0001\right)$.

ed from a polyfunctional to a more monofunctional response. The dominant immune response to TCR engagement caused $\mathrm{CD} 4^{+} \mathrm{T}$ cells to produce TNF- $\alpha$, a factor associated with dysfunctional stress responses. It has also been observed in malignancy that mediators released in reaction to stress suppress both specific and nonspecific parts of the immune system, production of cytokines, and cytotoxic $\mathrm{T}$ cell activity (49). As in our study, the effector responses associated with MIP-1 $\beta$ and IL-2 production were significantly reduced. In future studies it would be prudent to compare the cytokine secretion profile of treated individuals to HIV-infected, treatment-naive individuals to investigate whether the dysfunctionality evident in untreated HIV infection is restored by an INSTI-based ART regimen rather than other treatment regimens. The current findings are consistent with the observation of increased ROS production, which has previously been associated with $\mathrm{TNF}-\alpha$ production in $\mathrm{CD} 4^{+} \mathrm{T}$ cells.

Our data suggest a substantial interference of INSTIs with $\mathrm{CD} 4^{+} \mathrm{T}$ cell metabolism and function, yet this is in contrast with clinical experiences. Many previous studies have demonstrated safety and good efficacy of INSTIs in pediatric, adolescent, as well as adult individuals with rapid virological suppression compared with individuals on NNRTI- or PI-based regimens (50, 51). Although clinical trials analyze outcomes for patients primarily in terms of drug efficacy and adverse event profile, we have discovered that some drug side effects can be detected only by focused analysis of cellular biochemical mechanisms. However, the long-term clinical outcomes still need to be determined.

In summary, our study provides evidence of disruption in metabolic activity of lymphocytes during chronic, untreated HIV infection. We showed that the metabolic profile can be restored after ART initiation; however, 2 INSTIs, DLG and EVG, diminish the metabolic activity in $\mathrm{CD}^{+} \mathrm{T}$ cells, leading to a switch in functionality and impairment of overall function. Although the clinical relevance of these findings is unclear, future cohort studies where INSTI-based regimens are used over many years would be warranted to determine potential long-term toxicity. To this point, a recent study in Botswana reported that $0.9 \%$ of infants born to women receiving DLG-containing regimens had neural tube defects, while neural tube defects were observed only in $0.1 \%$ of infants born to women treated with another agent (52). Research is needed to understand the mechanism of action involved in this observation and should include studies of mitochondrial dysfunction.

\section{Methods}

Study population. Subsets of HIV-infected, treatment-naive $(n=11)$ and HIV-infected, treatment-experienced participants $(n=12)$ were recruited from the outpatient HIV and sexually transmitted disease clinic at the University Hospital Essen. HIV-uninfected specimens were obtained from the blood donation center.

Isolation of PBMCs. EDTA-containing blood samples were obtained from healthy donors and HIV-positive-treated and treatment-naive individuals, and blood mononuclear cells were isolated by standard density gradient protocol and cryopreserved as previously described (53). 
Cell subset separation and stimulation conditions. Cryopreserved PBMCs were thawed in R10 media (RPMI-1640 supplemented with 10\% heat-inactivated FCS, $2 \mathrm{mM}$ L-glutamine, $100 \mathrm{U} / \mathrm{mL}$ penicillin, 100 $\mu \mathrm{g} / \mathrm{mL}$ streptomycin, and $10 \mathrm{mM} \mathrm{HEPES}$ ) and allowed to rest overnight at $37^{\circ} \mathrm{C}, 5 \% \mathrm{CO}_{2}$. Different cell types were subsequently enriched by magnetic separation using MultiSort Isolation Kits (Miltenyi Biotec), and more than $90 \%$ purity was achieved. Cells were then stimulated using anti-CD3/CD28 beads (Gibco, Life Technologies) at a cell/bead ratio of $1: 1$ in the presence of IL-2 $(30 \mathrm{U} / \mathrm{mL})$ (eBioscience) for 3 days. Cells were cultured in R10 media in a humidified atmosphere $\left(5 \% \mathrm{CO}_{2}\right)$ at $37^{\circ} \mathrm{C}$. To assess the effect of antiretroviral medications, we incubated $\mathrm{CD} 4^{+} \mathrm{T}$ cells in the presence of different ART regimens - emtricitabine (FTC), zidovudine (AZT), tenofovir (TDF), lamivudine (3TC), rilpivirine (RLP), raltegravir (RAL), dolutegravir (DLG), elvitegravir (EVG), darunavir ethanolate (DRV), and ritonavir (RTV) (Selleckchem). Concentrations of the antiretroviral medications for each analysis are described in Supplemental Table 3.

Metabolic assay. OCR and ECAR were determined using a Seahorse XFe Extracellular Flux Analyzer according to manufacturer's protocol (54). Briefly, cells were plated on a poly-D-lysine-coated, 8-well polystyrene Seahorse plate at a density of 200,000 cells per well. Cells were equilibrated in unbuffered DMEM supplemented with $2 \mathrm{mM}$ glutamine, $2 \mathrm{mM}$ sodium pyruvate, and $10 \mathrm{mM}$ glucose for $45 \mathrm{~min}$ utes at $37^{\circ} \mathrm{C}$ with $0 \% \mathrm{CO}_{2}$ atmosphere before the experiment. Metabolic assays were performed using the following concentrations of subsequently injected compounds: oligomycin $1 \mu \mathrm{M}$, mitochondrial uncoupler phosphorylated trifluormethoxy carbonyl cyanice phenol hydrazine (FCCP) $1 \mu \mathrm{M}$, and antimycin A and rotenone (both $0.5 \mu \mathrm{M}$ ). At each time interval, the OCR and ECAR values were measured as indicators of mitochondrial respiration and glycolysis, respectively.

Phenotypic assessment by flow cytometry. Cryopreserved PBMCs were thawed and rested overnight as described above. We passed 1,000,000 PBMCs from each individual through a $40-\mu \mathrm{m}$ cell strainer (BD Falcon) to obtain single-cell suspensions. Cells were washed with PBS and stained with viability dye Zombie Aqua (BioLegend). Cells were subsequently washed with staining buffer (PBS supplemented with $2 \%$ FCS) and stained with fluorescently conjugated antibodies: anti-CD3-AF488 (clone UCHT1; BioLegend), anti-CD4-AF700 (clone RPA-T4; BioLegend), anti-CD8-APC-Cy7 (clone RPA-T8; BioLegend), anti-CD38-BV605 (clone HIT2; BioLegend), anti-HLA-DR-APC (clone L243; BioLegend), and anti-PD-1-PE (clone EH12.2h7 BioLegend). Data were collected on an FACSAria III (BD Biosciences) and analyzed with FlowJo software version 10.1 (Tree Star).

Assessment of ex vivo proliferation by flow cytometry. Cryopreserved PBMCs from HIV-positive, treated individuals were thawed and rested overnight as described above. Afterward, approximately 3,000,000 PBMCs from each individual were washed with PBS and stained with the cell division tracker carboxyfluorescein diacetate succinimidyl ester (CFSE, BioLegend). Thereafter, cells were resuspended in $1 \mathrm{~mL}$ PBS and stained with $1.25 \mu \mathrm{M} \mathrm{CFSE}$ at $37^{\circ} \mathrm{C}, 5 \% \mathrm{CO}_{2}$, for 20 minutes. Subsequently, the staining was quenched by adding 5 times the original staining volume of R10 media. Cells from each individual were centrifuged, resuspended in R10 media, and transferred to a 24 -well polystyrene plate (Starlab) at a concentration of 1,000,000 million/mL. Cells were stimulated with $5 \mu \mathrm{g} / \mathrm{mL}$ SEB or left unstimulated as a control at $37^{\circ} \mathrm{C}$, $5 \% \mathrm{CO}_{2}$, for 5 days. On the fifth day, cells were washed with PBS and stained with viability dye Zombie Aqua (BioLegend) for 30 minutes. After washing with staining buffer (PBS supplemented with 2\% FCS), cells were stained extracellularly for 20 minutes with fluorescently conjugated antibodies: anti-CD3-APCCy7 (clone UCHT1; BioLegend), anti-CD4-BV421 (clone RPA-T4; BioLegend), and anti-CD8-AF647 (clone C8/144B; BioLegend). Data were collected on an FACSCelesta (BD Biosciences) and analyzed with the proliferation tool of FlowJo software version 10.1 (Tree Star).

Assessment of T cell polyfunctionality. Cryopreserved PBMCs isolated from healthy donors were thawed and rested overnight as described above. Cells were transferred to a 24 -well polystyrene plate (Starlab) at a concentration of $1,000,000 / \mathrm{mL}$ and stimulated with $200 \mathrm{ng} / \mathrm{mL}$ SEB in the presence of different ART regimens at $37^{\circ} \mathrm{C}, 5 \% \mathrm{CO}_{2}$, for 3 days. On day 3, cells were restimulated with $5 \mu \mathrm{g} / \mathrm{mL} \mathrm{SEB}$ and incubated in the presence of costimulatory molecule CD28/CD49d (BD Biosciences) with antiCD107a-PE-Cy5 (clone H4A3; BD Biosciences) for 6 hours. After 1 hour, Golgi Stop and Golgi Plug (BD Biosciences) were added, and cells were incubated for an additional 5 hours. Cells were then washed with PBS and stained with viability dye Zombie Aqua (BioLegend). After washing with staining buffer (PBS supplemented with 2\% FCS), CytoFix/CytoPerm Solution (BD Biosciences) was added, and cells were incubated for 20 minutes at $4^{\circ} \mathrm{C}$. Cells were then washed with $1 \times$ Perm/Wash Buffer (BD Biosciences) and stained intracellularly with fluorescently conjugated antibodies: anti-CD3 Pacific Blue 
(clone UCHT1; BioLegend), anti-CD4-BV421 (clone RPA-P4; BioLegend), anti-CD8-APC-Cy7 (clone RPA-T8; BioLegend), anti-MIP-1 $\beta$ PE (clone D21-1351, BD Biosciences), anti-TNF- $\alpha$ AF700 (clone Mab11; BioLegend), anti-IFN- $\gamma$ PECy7 (clone B27; BioLegend), and FITC anti-IL-2 (clone MQ117H12; BioLegend). Data were collected and analyzed as above.

Protein isolation and HIF-1 $\alpha$ immunoblot. $\mathrm{CD}^{+} \mathrm{T}$ cells were activated in the presence of an NRTI (FTC), NNRTI (RLP), INSTI (DLG), and PI (DRV) for 3 days. Hypoxic incubation was performed in a Ruskinn Invivo2 300 workstation under $1 \% \mathrm{O}_{2}$ for 4 hours. Cells were lysed under normoxic or hypoxic conditions with a common immunoblot lysis buffer (150 mM NaCl, $20 \mathrm{mM}$ Tris at pH 7.5, 1\% NP-40, 5 mM EDTA) containing $10 \%$ freshly added proteinase inhibitor (Roche) for 20 minutes on wet ice. Lysates were centrifuged at $1452 \mathrm{~g}, 4^{\circ} \mathrm{C}$ for 5 minutes, in a microcentrifuge, and supernatants containing cellular proteins were collected and stored at $-80^{\circ} \mathrm{C}$. Protein concentration was measured with the Bio-Rad DC Protein Assay. We subjected $50 \mu \mathrm{g}$ of whole-cell lysate to $7.5 \%$ SDS-PAGE and transferred it onto a nitrocellulose membrane $(0.2-\mu \mathrm{M}$ pore size; Schleicher \& Schuell Microscience). HIF-1 $\alpha$ protein was detected by a monoclonal anti-HIF-1 $\alpha$ antibody (BD Transduction; 610958), and $\beta$-actin (MilliporeSigma; A2103-200UL) served as a loading control.

$R N A$ isolation and gene expression analysis by quantitative PCR. CD4 ${ }^{+} \mathrm{T}$ cells were treated as indicated for immunoblot. Total RNA was isolated by the acid guanidinium thiocyanate-phenol-chloroform extraction method (55). We reverse-transcribed $1 \mu \mathrm{g}$ of total RNA into cDNA, and performed quantification of HIF target genes by real-time PCR (SYBR Green PCR reaction with Blue S'Green qPCR Kit from Biozym, with Bio-Rad CFX 96 Touch qPCR system). The primer sequences used for qualitative and quantitative PCR are listed in Supplemental Table 4. Data were analyzed with $\Delta \Delta C t$ method and are indicated as fold change over DMSO-treated control.

Assessment of cell viability. Viability of cells was repeatedly assessed by using trypan blue staining. Briefly, $10 \mu \mathrm{L}$ of cell suspension was mixed with $10 \mu \mathrm{L}$ of $0.4 \%$ trypan blue and loaded onto a cell counting slide. Afterward, the number of live cells was determined using an automatic cell counter EVE (NanoEnTek). An exception was made for the NNRTI efavirenz, which showed cytotoxicity at low plasma concentrations in vitro and was therefore excluded from these studies. For the determination of whether the living cells undergo early apoptosis, cells were stained repeatedly with annexin V. Cells were washed with staining buffer (PBS supplemented with 2\% FCS) and stained extracellularly for 20 minutes with fluorescently conjugated antibodies anti-CD3-APC-Cy7 (clone UCHT1; BioLegend) and anti-CD4-BV421 (clone RPA-T4; BioLegend). Afterward, cells were washed with Annexin V Binding Buffer (BioLegend) and stained with anti-FITC annexin V (catalog 640914, BioLegend) antibody for 15 minutes. Subsequently, $400 \mu \mathrm{L}$ of Annexin V Binding Buffer was added to the cells. Data were collected on an FACSCelesta (BD Biosciences) and analyzed with FlowJo software version 10.1 (Tree Star).

Measuring mtROS. To detect mtROS, MitoSox red mitochondrial superoxide indicator (Thermo Fisher Scientific) was used according to the manufacturer's protocol. Shortly afterward, cells were washed with warm Hanks' balanced salt solution with calcium and magnesium (HBBS/Ca/Mg) and resuspended in $1 \mathrm{~mL}$ of 5 - $\mu \mathrm{M}$ MitoSox reagent (supplied with the kit). Cells were incubated at $37^{\circ} \mathrm{C}$, $5 \% \mathrm{CO}_{2}$, for 10 minutes. Afterward, cells were washed 3 times with warm $\mathrm{HBBS} / \mathrm{Ca} / \mathrm{Mg}$. Data were collected and analyzed as above.

$m t D N A$ content. Total cellular DNA was isolated using a DNA mini isolation kit (Qiagen). Real-time PCR was performed using the thermal cycler qTower 2.0 (Analytic Jena) with innuMIX qPCR MasterMix SyGreen (Analytic Jena) and mtDNA content assessed as previously described (56).

Assessment of biological antiviral activity in vitro. Cryopreserved PBMCs isolated from healthy, HIV-negative donors were thawed, rested overnight, and activated with $1 \mu \mathrm{g} / \mathrm{mL}$ of PHA (Sigma Aldrich) for 3 days. They were then seeded in a 6-well polystyrene plate (Star Lab) at a density of $1.5 \times 10^{6}$ cells $/ \mathrm{mL}$ and infected with $67 \mathrm{ng} / \mathrm{mL}$ of HIV-1 US-1 virus (National Institute of Allergy and Infectious Diseases [NIH], AIDS Reagents Program). Different ART regimens at 5-fold plasma concentration (DLG, RAL, and EVG as well as TDF and TFV) were added into the suspension, and cells were incubated at $37^{\circ} \mathrm{C}$, $5 \% \mathrm{CO}_{2}$, for another 3 or 4 days. Cells were harvested, washed with PBS, and stained with viability dye Zombie Aqua (BioLegend) for 30 minutes at room temperature. Cells were subsequently washed with staining buffer (PBS supplemented with $2 \%$ FCS) and stained with fluorescently conjugated antibody anti-CD8-AF647 (clone RPA-T8; BioLegend) for 20 minutes at $4^{\circ} \mathrm{C}$. For intercellular staining, cells were washed again with staining buffer and incubated with CytoFix/CytoPerm Solution (BD Biosciences) for another 20 minutes at $4^{\circ} \mathrm{C}$. After being washed with $1 \times$ Perm/Wash Buffer (BD Biosciences), cells were 
intracellularly stained with fluorescently conjugated antibodies anti-CD3-APC-Cy7 (clone UCHT1; BioLegend), anti-CD4-BV421 (clone RPA-P4; BioLegend), and anti-p24-PE (clone KC57; Beckman Coulter) for 30 minutes at $20^{\circ} \mathrm{C}$. Data were collected on a BD Biosciences FACSCelesta and analyzed with FlowJo (version 10.1) as described above.

Availability of data. The authors confirm that all data underlying the findings are fully available. Clinical and experimental data can be requested from Hendrik Streeck at the Institute for HIV Research, University Duisburg-Essen.

Statistics. Prism (GraphPad Software) was used for statistical analysis. Data are presented as mean \pm SD for technical replicates or mean \pm SEM for biological replicates. Differences between treatment categories were estimated with 1-way ANOVA. $P<0.05$ indicates statistical significance.

Study approval. None of the funding sources were involved in the design or carrying out of the study. The study was approved by the IRB at the University Duisburg-Essen. Informed consent was obtained from all participants involved in the study.

\section{Author contributions}

MK, ER, BTS, PJ, BDLR, and SW performed, analyzed, and optimized laboratory work. MK, ER, PJ, SE, $\mathrm{BKA}$, and $\mathrm{HS}$ were involved in patient data collection. $\mathrm{MK}, \mathrm{MB}$, and BKA were involved in statistical evaluation. HS wrote the first draft of the manuscript. MK, MB, ER, BTS, PJ, JAA, AG, JFO, MLR, BDLR, SW, JF, THB, SE, NLM, BKA, and HS provided edits, discussion, and changes of the manuscript.

\section{Acknowledgments}

This project has been funded in whole, or in part, by the German Research Foundation (DFG). This project has been funded in part with federal funds from the National Institute of Allergy and Infectious Diseases, NIH, under Inter-Agency Agreement Y1-AI-5072. Portions of this study were also supported by the Infectious Disease Clinical Research Program, a Department of Defense program executed through the Uniformed Services University of the Health Sciences. The views expressed are those of the authors and should not be construed to represent the positions of the United States Departments of the Navy, Army, or Defense; the Uniformed Services University of the Health Sciences; or the Henry M. Jackson Foundation for the Advancement of Military Medicine.

Address correspondence to: Hendrik Streeck, Institute for HIV Research, Universitätsklinikum Essen, Virchowstr. 171, 45147 Essen, Germany. Phone: 49.201.723.4225; Email: hendrik.streeck@uk-essen.de.

1. Khaitan A, Unutmaz D. Revisiting immune exhaustion during HIV infection. Curr HIV/AIDS Rep. 2011;8(1):4-11.

2. Paiardini M, Müller-Trutwin M. HIV-associated chronic immune activation. Immunol Rev. 2013;254(1):78-101.

3. Chang CH, et al. Posttranscriptional control of T cell effector function by aerobic glycolysis. Cell. 2013;153(6):1239-1251.

4. Schmidt EA, et al. Metabolic alterations contribute to enhanced inflammatory cytokine production in Irgm1-deficient macrophages. J Biol Chem. 2017;292(11):4651-4662.

5. Delgoffe GM, Powell JD. Feeding an army: The metabolism of T cells in activation, anergy, and exhaustion. Mol Immunol. 2015;68(2 pt C):492-496.

6. Blagih J, et al. The energy sensor AMPK regulates $\mathrm{T}$ cell metabolic adaptation and effector responses in vivo. Immunity. 2015;42(1):41-54

7. Warburg O. On respiratory impairment in cancer cells. Science. 1956;124(3215):269-270.

8. Sharpe AH, Wherry EJ, Ahmed R, Freeman GJ. The function of programmed cell death 1 and its ligands in regulating autoimmunity and infection. Nat Immunol. 2007;8(3):239-245.

9. Bengsch B, et al. Bioenergetic insufficiencies due to metabolic alterations regulated by the inhibitory receptor PD-1 are an early driver of CD8(+) T cell exhaustion. Immunity. 2016;45(2):358-373.

10. Patsoukis N, et al. PD-1 alters T-cell metabolic reprogramming by inhibiting glycolysis and promoting lipolysis and fatty acid oxidation. Nat Commun. 2015;6:6692.

11. Streeck H, et al. Antigen load and viral sequence diversification determine the functional profile of HIV-1-specific CD8 ${ }^{+} \mathrm{T}$ cells PLoS Med. 2008;5(5):e100.

12. Arts EJ, Hazuda DJ. HIV-1 antiretroviral drug therapy. Cold Spring Harb Perspect Med. 2012;2(4):a007161.

13. Battegay M, Elzi L. Does HIV antiretroviral therapy still need its backbone? Lancet. 2014;384(9958):1908-1910.

14. Hazuda DJ. HIV integrase as a target for antiretroviral therapy. Curr Opin HIV AIDS. 2012;7(5):383-389.

15. Clotet B, et al. Once-daily dolutegravir versus darunavir plus ritonavir in antiretroviral-naive adults with HIV-1 infection (FLAMINGO): 48 week results from the randomised open-label phase 3b study. Lancet. 2014;383(9936):2222-2231.

16. Lennox JL, et al. Safety and efficacy of raltegravir-based versus efavirenz-based combination therapy in treatment-naive patients with HIV-1 infection: a multicentre, double-blind randomised controlled trial. Lancet. 2009;374(9692):796-806. 
17. Zolopa AR, et al. Activity of elvitegravir, a once-daily integrase inhibitor, against resistant HIV Type 1: results of a phase 2, randomized, controlled, dose-ranging clinical trial. J Infect Dis. 2010;201(6):814-822.

18. Raffi F, et al. Once-daily dolutegravir versus raltegravir in antiretroviral-naive adults with HIV-1 infection: 48 week results from the randomised, double-blind, non-inferiority SPRING-2 study. Lancet. 2013;381(9868):735-743.

19. Günthard HF, et al. Antiretroviral drugs for treatment and prevention of HIV infection in adults: 2016 Recommendations of the International Antiviral Society-USA Panel. JAMA. 2016;316(2):191-210.

20. Troya J, Bascuñana J. Safety and tolerability: current challenges to antiretroviral therapy for the long-term management of HIV infection. AIDS Rev. 2016;18(3):127-137.

21. White AJ. Mitochondrial toxicity and HIV therapy. Sex Transm Infect. 2001;77(3):158-173.

22. Masson JJR, Murphy AJ, Lee MKS, Ostrowski M, Crowe SM, Palmer CS. Assessment of metabolic and mitochondrial dynamics in $\mathrm{CD}^{+}$and $\mathrm{CD} 8^{+} \mathrm{T}$ cells in virologically suppressed HIV-positive individuals on combination antiretroviral therapy. PLoS One. 2017;12(8):e0183931.

23. Samaras K. Metabolic consequences and therapeutic options in highly active antiretroviral therapy in human immunodeficiency virus-1 infection. J Antimicrob Chemother. 2008;61(2):238-245.

24. Maganga E, et al. Glucose metabolism disorders, HIV and antiretroviral therapy among Tanzanian adults. PLoS One. 2015;10(8):e0134410.

25. Emadi A, Ross AE, Cowan KM, Fortenberry YM, Vuica-Ross M. A chemical genetic screen for modulators of asymmetrical 2,2'-dimeric naphthoquinones cytotoxicity in yeast. PLoS One. 2010;5(5):e10846.

26. Kestens L, et al. Expression of activation antigens, HLA-DR and CD38, on CD8 lymphocytes during HIV-1 infection. AIDS. 1992;6(8):793-797.

27. Zoratto E, Norelli MT, Malvestio G, Ferrero AM, Cerutti A. [Study of the relation between blood iron and HbA2 synthesis in infants]. Minerva Pediatr. 1978;30(7):555-566.

28. Wherry EJ, Blattman JN, Murali-Krishna K, van der Most R, Ahmed R. Viral persistence alters CD8 T-cell immunodominance and tissue distribution and results in distinct stages of functional impairment. $J$ Virol. 2003;77(8):4911-4927.

29. Ferre AL, et al. HIV controllers with HLA-DRB1*13 and HLA-DQB1*06 alleles have strong, polyfunctional mucosal CD4 ${ }^{+}$ T-cell responses. J Virol. 2010;84(21):11020-11029.

30. Emu B, et al. Phenotypic, functional, and kinetic parameters associated with apparent T-cell control of human immunodeficiency virus replication in individuals with and without antiretroviral treatment. J Virol. 2005;79(22):14169-14178.

31. Teigler JE, et al. Differential inhibitory receptor expression on $\mathrm{T}$ cells delineates functional capacities in chronic viral infection. J Virol. 2017;91(23):e01263-17.

32. Sato M, et al. Novel HIV-1 integrase inhibitors derived from quinolone antibiotics. JMed Chem. 2006;49(5):1506-1508.

33. Suto MJ, Domagala JM, Roland GE, Mailloux GB, Cohen MA. Fluoroquinolones: relationships between structural variations, mammalian cell cytotoxicity, and antimicrobial activity. J Med Chem. 1992;35(25):4745-4750.

34. Lewis W, Dalakas MC. Mitochondrial toxicity of antiviral drugs. Nat Med. 1995;1(5):417-422.

35. Renner K, et al. Metabolic plasticity of human T cells: Preserved cytokine production under glucose deprivation or mitochondrial restriction, but 2-deoxy-glucose affects effector functions. Eur J Immunol. 2015;45(9):2504-2516.

36. Aounallah M, et al. Current topics in HIV pathogenesis, part 2: Inflammation drives a Warburg-like effect on the metabolism of HIV-infected subjects. Cytokine Growth Factor Rev. 2016;28:1-10.

37. Datta PK, et al. Glutamate metabolism in HIV-1 infected macrophages: role of HIV-1 Vpr. Cell Cycle. 2016;15(17):2288-2298.

38. Palmer CS, Ostrowski M, Balderson B, Christian N, Crowe SM. Glucose metabolism regulates T cell activation, differentiation, and functions. Front Immunol. 2015;6:1.

39. Streeck H, et al. Immunological and virological impact of highly active antiretroviral therapy initiated during acute HIV-1 infection. J Infect Dis. 2006;194(6):734-739.

40. Rajasuriar R, Wright E, Lewin SR. Impact of antiretroviral therapy (ART) timing on chronic immune activation/inflammation and end-organ damage. Curr Opin HIV AIDS. 2015;10(1):35-42.

41. Elemans M, Seich Al Basatena NK, Klatt NR, Gkekas C, Silvestri G, Asquith B. Why don't CD8 ${ }^{+}$T cells reduce the lifespan of SIV-infected cells in vivo? PLoS Comput Biol. 2011;7(9):e1002200.

42. Mudd JC, Lederman MM. CD8 T cell persistence in treated HIV infection. Curr Opin HIV AIDS. 2014;9(5):500-505.

43. Ford N, et al. The WHO public health approach to HIV treatment and care: looking back and looking ahead. Lancet Infect Dis. 2018;18(3):e76-e86.

44. Negredo E, et al. Early but limited effects of raltegravir intensification on CD4 T cell reconstitution in HIV-infected patients with an immunodiscordant response to antiretroviral therapy. J Antimicrob Chemother. 2013;68(10):2358-2362.

45. Wallace ZR, Sanderson S, Simon AK, Dorrell L. Exposure to zidovudine adversely affects mitochondrial turnover in primary T cells. Antiviral Res. 2016;133:178-182.

46. Lewis W. Mitochondrial dysfunction and nucleoside reverse transcriptase inhibitor therapy: experimental clarifications and persistent clinical questions. Antiviral Res. 2003;58(3):189-197.

47. Gerschenson M, Brinkman K. Mitochondrial dysfunction in AIDS and its treatment. Mitochondrion. 2004;4(5-6):763-777.

48. Kalghatgi S, et al. Bactericidal antibiotics induce mitochondrial dysfunction and oxidative damage in Mammalian cells. Sci Transl Med. 2013;5(192):192ra85.

49. Reiche EM, Nunes SO, Morimoto HK. Stress, depression, the immune system, and cancer. Lancet Oncol. 2004;5(10):617-625.

50. Jacobson K, Ogbuagu O. Integrase inhibitor-based regimens result in more rapid virologic suppression rates among treatment-naïve human immunodeficiency virus-infected patients compared to non-nucleoside and protease inhibitor-based regimens in a real-world clinical setting: A retrospective cohort study. Medicine (Baltimore). 2018;97(43):e13016.

51. Bruzzese E, et al. Dolutegravir-based anti-retroviral therapy is effective and safe in HIV-infected paediatric patients. Ital J Pediatr. 2018;44(1):37.

52. World Health Organization. Potential safety issue affecting women living with HIV using dolutegravir at the time of conception. WHO Web Site. http://www.who.int/medicines/publications/drugalerts/Statement_on_DTG_18May_2018final.pdf. Published May 18, 2018. Accessed May 22, 2019. 
53. Streeck H, et al. Recognition of a defined region within p24 gag by CD8 ${ }^{+} \mathrm{T}$ cells during primary human immunodeficiency virus type 1 infection in individuals expressing protective HLA class I alleles. J Virol. 2007;81(14):7725-7731.

54. Pelletier M, Billingham LK, Ramaswamy M, Siegel RM. Extracellular flux analysis to monitor glycolytic rates and mitochondrial oxygen consumption. Meth Enzymol. 2014;542:125-149.

55. Chomczynski P, Sacchi N. Single-step method of RNA isolation by acid guanidinium thiocyanate-phenol-chloroform extraction. Anal Biochem. 1987;162(1):156-159.

56. Venegas V, Halberg MC. Measurement of mitochondrial DNA copy number. Methods Mol Biol. 2012;837:327-335 